\title{
On a Characterization of Compact Hausdorff Space $X$ for Which Certain Algebraic Equations Are Solvable in $C(X)$
}

\author{
Dai HONMA and Takeshi MIURA \\ Niigata University and Yamagata University \\ (Communicated by K. Taniyama)
}

\begin{abstract}
Let $X$ be a compact Hausdorff space and $C(X)$ the Banach algebra of all complex-valued continuous functions on $X$. We consider the following property of $C(X)$ : for each $f \in C(X)$ there exist a $g \in C(X)$ and positive integers $p$ and $q$ such that $p$ does not divide $q$ and $f^{q}=g^{p}$. When $X$ is locally connected, we give a necessary and sufficient condition for $C(X)$ to have this property. We also give a characterization of a first-countable compact Hausdorff space $X$ for which $C(X)$ has the property above. As a corollary, we prove that if $X$ is locally connected, or first-countable, then $C(X)$ has the property above if and only if $C(X)$ is algebraically closed.
\end{abstract}

\section{Introduction and the statement of results}

Let $X$ be a compact Hausdorff space and $C(X)$ the Banach algebra of all complex-valued continuous functions on $X$ with respect to the pointwise operations and the supremum norm $\|\cdot\|_{\infty}$. Suppose that $X$ is locally connected and $A$ is a uniform algebra on $X$. Cirka [2] proved that if to each $f \in A$ there corresponds a $g \in A$ such that $f=g^{2}$, then $A=C(X)$. On the other hand, there is no continuous function on the unit circle $S^{1}$ in the complex plane C, whose square is the identity function on $S^{1}$. Hatori and Miura [8, Theorem 2.2] gave a characterization in order for $C(X)$ to be square root closed, that is, to each $f \in C(X)$ there corresponds a $g \in C(X)$ such that $f=g^{2}$. To be more explicit, $C(X)$ is square root closed if and only if the covering dimension of $X$ is less than or equal to 1 and the first Čech cohomology group with integer coefficient is trivial.

Let $P(x, z)$ be a monic polynomial over $C(X)$ : for a positive integer $n$ and $a_{0}, a_{1}, \ldots, a_{n-1} \in C(X), P(x, z)=z^{n}+a_{n-1}(x) z^{n-1}+\cdots+a_{1}(x) z+a_{0}(x)$ for $x \in X$. We say that $C(X)$ is algebraically closed if for each monic polynomial $P(x, z)$ over $C(X)$ there exists an $f \in C(X)$ such that $P(x, f(x))=0$ for every $x \in X$. By definition, $C(X)$ is square root closed if $C(X)$ is algebraically closed. Deckard and Pearcy $[4,5]$ proved that $C(X)$ is algebraically closed if $X$ is a Stonian space, or a totally disconnected compact Hausdorff space, or a linearly ordered and order-complete topological space. They also remarked that if $X$ is the closure of the graph of the function $y=\sin 1 / x, 0<x \leq 1$, then there exists

2000 Mathematics Subject Classification: 46J10.

Key words and Phrases: commutative $C^{*}$-algebras, maximal ideal spaces 
a continuous function $f$ of $X$ into $\mathbf{C}$ such that $f \neq g^{2}$ for any $g \in C(X)$. Countryman, Jr. [3] gave some necessary and sufficient conditions for a first-countable compact Hausdorff space $X$ in order that $C(X)$ is algebraically closed. For example, $C(X)$ is algebraically closed if and only if $C(X)$ is square root closed. Moreover, for every first-countable space, these are also equivalent to the condition that $X$ is hereditarily unicoherent and almost locally connected. Miura and Niijima [13] gave some necessary and sufficient condition for a locally connected compact Hausdorff space $X$ in order for $C(X)$ be algebraically closed.

It seems that Gorin and Karahanjan [7] strengthened the above result of Čirka as follows: If $A$ is a uniform algebra on a locally connected compact Hausdorff space $X$ with the property that for each $f \in A$ there exist a $g \in A$ and a $p \in \mathbf{N}, p \geq 2$ such that $f=g^{p}$, then $A=C(X)$. Furthermore, Karahanjan (cf. [9, Theorem 1]) weakened the hypothesis in the following way and proved that $A=C(X)$ whenever $X$ is locally connected:

(*) For every $f \in A$ there exist a $g \in A$ and $p, q \in \mathbf{N}$ such that $q / p \notin \mathbf{N}$ and $f^{q}=g^{p}$.

Note that if we replace " $q / p \notin \mathbf{N}$ " with " $q / p \in \mathbf{N}$ " in (*), then the condition $(*)$ obviously holds for every $A$.

In this paper, we give a necessary and sufficient condition for a locally connected compact Hausdorff space $X$ in order that $C(X)$ satisfies the condition (*). As a corollary, we also prove that if $X$ is locally connected, or first-countable, then the condition (*) holds for $C(X)$ if and only if $C(X)$ is algebraically closed; In this case, $(*)$ for $C(X)$ is equivalent to the square root closedness of $C(X)$.

We say that a topological space $T$ is almost locally connected if $T$ contains no mutually disjoint connected closed subsets $C_{n}(n \in \mathbf{N})$, which are open in the closure of $\cup_{n \in \mathbf{N}} C_{n}$ in $T$, with the following property: There exist $x_{n}, y_{n} \in C_{n}$ such that $\left\{x_{n}\right\}_{n \in \mathbf{N}}$ and $\left\{y_{n}\right\}_{n \in \mathbf{N}}$ converge to distinct points. For example, the closure of the graph of the function $y=\sin 1 / x$, $0<x \leq 1$ is not almost locally connected.

We say that a topological space $T$ is hereditarily unicoherent if $M \cap N$ is connected for every pair of closed connected subsets $M$ and $N$ of $T$. For example, the unit circle $S^{1}$ is not hereditarily unicoherent.

Let $Y$ be a normal space and $n$ a non-negative integer. The covering dimension $\operatorname{dim} Y$ of $Y$ is less than or equal to $n$ if for every finite open covering $\mathfrak{A}$ of $Y$ there exists a refinement $\mathfrak{B}$ of $\mathfrak{A}$ such that each $y \in Y$ belongs to at most $(n+1)$ elements of $\mathfrak{B}$. It is well-known that $\operatorname{dim} Y \leq n$ if and only if for every closed subset $F$ of $Y$ and every $S^{n}$-valued continuous function $f$ on $F$, there exists an $S^{n}$-valued continuous function $\tilde{f}$ on $Y$ such that $\left.\tilde{f}\right|_{F}=f$, where $S^{n}$ is the $n$-sphere (cf. [14]).

Let $X$ be a compact Hausdorff space. Then $\check{H}^{1}(X ; \mathbf{Z})$ denotes the first Čech cohomology group of $X$ with integer coefficients. Let $C(X)^{-1}$ be the multiplicative group of all invertible elements of $C(X)$ and $\exp C(X)=\left\{e^{f}: f \in C(X)\right\}$. It is well-known that $\check{H}^{1}(X ; \mathbf{Z})$ is isomorphic to the quotient group $C(X)^{-1} / \exp C(X)$, by a theorem of Arens and Royden [6]. In particular, $\check{H}^{1}(X ; \mathbf{Z})$ is trivial if and only if $C(X)^{-1}=\exp C(X)$.

Now we are ready to state our main result. The main result of this paper is as follows: 
THEOREM 1.1. Let X be a locally connected compact Hausdorff space. Then the following conditions are equivalent.

(a) For each $f \in C(X)$ there exist $p, q \in \mathbf{N}$ and $g \in C(X)$ such that $q / p \notin \mathbf{N}$ and $f^{q}=g^{p}$.

(b) $X$ is hereditarily unicoherent.

(c) $\operatorname{dim} X \leq 1$ and $\check{H}^{1}(X ; \mathbf{Z})$ is trivial.

(d) $\left\{g^{p}: g \in C(X)\right\}$ is uniformly dense in $C(X)$ for every $p \in \mathbf{N}$.

(e) For each $f \in C(X)$ and $p \in \mathbf{N}$ there exists a $g \in C(X)$ such that $f=g^{p}$.

COROLlaRY 1.2. Let X be a locally connected compact Hausdorff space. Then the following conditions are equivalent.

(a) For each $f \in C(X)$ there exist $p, q \in \mathbf{N}$ and $g \in C(X)$ such that $q / p \notin \mathbf{N}$ and $f^{q}=g^{p}$.

(b) $\left\{g^{p}: g \in C(X)\right\}$ is uniformly dense in $C(X)$ for every $p \in \mathbf{N}$.

(c) For each $f \in C(X)$ and $p \in \mathbf{N}$ there exists a $g \in C(X)$ such that $f=g^{p}$.

(d) $C(X)$ is algebraically closed.

(e) $C(X)$ is square-root closed.

(f) $X$ is hereditarily unicoherent.

(g) $\operatorname{dim} X \leq 1$ and $\check{H}^{1}(X ; \mathbf{Z})$ is trivial.

COROLlary 1.3. Let X be a first-countable compact Hausdorff space. Then each of the following conditions implies the other.

(a) For each $f \in C(X)$ there exist $p, q \in \mathbf{N}$ and $g \in C(X)$ such that $q / p \notin \mathbf{N}$ and $f^{q}=g^{p}$.

(b) $C(X)$ is algebraically closed.

(c) $C(X)$ is square-root closed.

(d) $X$ is hereditarily unicoherent and almost locally connected.

(e) $X$ is almost locally connected, $\operatorname{dim} X \leq 1$ and $\check{H}^{1}(X ; \mathbf{Z})$ is trivial.

\section{Lemmas}

We require some lemmas before proving Theorem 1.1. To prove Lemmas 2.1 and 2.2, we use ideas by Countryman, Jr. [3, Lemma 2.1, Lemma 2.3].

LEMMA 2.1. Let $X$ be a compact Hausdorff space. If the condition (a) of Theorem 1.1 holds, then $X$ is hereditarily unicoherent.

Proof. Assume that the condition (a) holds. We will show that $X$ is hereditarily unicoherent. Suppose not. Then, by definition, there exist non-empty closed connected subsets $M$ and $N$ of $X$ such that $M \cap N$ is disconnected. So, there are non-empty closed subsets $A$ and $B$ such that $M \cap N=A \cup B$ and $A \cap B=\emptyset$. Let $f$ be a continuous mapping of $X$ into 
the closed unit interval $[0,1]$ such that $f(x)=0$ on $A$ and $f(x)=1$ on $B$. Put

$$
h(x)= \begin{cases}\exp (i \pi f(x)) & x \in M \\ \exp (-i \pi f(x)) & x \in N \backslash M .\end{cases}
$$

Then we see that $h$ is continuous on $M \cup N$. Let $\tilde{h} \in C(X)$ be a mapping so that $\left.\tilde{h}\right|_{M \cup N}=h$. By the condition (a), there exist positive integers $p, q$ and an element $\tilde{g}$ in $C(X)$ such that $p$ does not divide $q$ and $\tilde{h}^{q}=\tilde{g}^{p}$. Put $q=s p+r$, where $s$ and $r$ are integers with $1 \leq r \leq p-1$ (note $q / p \notin \mathbf{N}$ ). Since $h$ does not vanish on $M \cup N$, the function $g=\left.\tilde{g}\right|_{M \cup N} / h^{s}$ is a welldefined continuous mapping of $M \cup N$ into $\mathbf{C}$. Since $\tilde{h}^{q}=\tilde{g}^{p}$, for each $x \in M \cup N$ we obtain

$$
g^{p}(x)=\left(\frac{\tilde{g}(x)}{h^{s}(x)}\right)^{p}=\frac{\tilde{h}^{q}(x)}{h^{s p}(x)}=h^{q-s p}(x)=h^{r}(x),
$$

and so $h^{r}=g^{p}$ on $M \cup N$. Since

$$
g^{p}(x)=h^{r}(x)=\exp (i \pi r f(x))
$$

for $x \in M$, we get

$$
g(x)=\omega(x) \exp \left(\frac{i \pi r f(x)}{p}\right)
$$

for every $x \in M$, where $\omega(x)$ is one of the $p$-th roots of 1 . The above equation and the continuity of $f$ and $g$ imply that $\omega(x)$ is a continuous mapping of $M$ into the set of all $p$-th roots of 1 . Since $M$ is connected, $\omega$ must be constant. So there is a $p$-th root $\omega_{0}$ of 1 such that

$$
g(x)=\omega_{0} \exp \left(\frac{i \pi r f(x)}{p}\right)
$$

for each $x$ in $M$. In a way similar to the above, we see that there exists a $p$-th root $\gamma_{0}$ of 1 such that

$$
g(x)=\gamma_{0} \exp \left(-\frac{i \pi r f(x)}{p}\right)
$$

for each $x$ in $N$.

Pick an $x_{0} \in A$ arbitrarily. Since $x_{0} \in A \subset M \cap N$, the equations (1) and (2) imply that

$$
\omega_{0} \exp \left(\frac{i \pi r f\left(x_{0}\right)}{p}\right)=g\left(x_{0}\right)=\gamma_{0} \exp \left(-\frac{i \pi r f\left(x_{0}\right)}{p}\right) .
$$

Recall that $f=0$ on $A$ and $f=1$ on $B$, and so $f\left(x_{0}\right)=0$. We thus obtain $\omega_{0}=\gamma_{0}$. For $y \in B$, it follows from (1), (2) and $\omega_{0}=\gamma_{0}$ that

$$
\omega_{0} \exp \left(\frac{i \pi r}{p}\right)=g(y)=\omega_{0} \exp \left(-\frac{i \pi r}{p}\right)
$$


because $B \subset M \cap N$. Thus we have $r / p \in \mathbf{N}$, which contradicts $1 \leq r<p-1$. We conclude that $X$ is hereditarily unicoherent.

Lemma 2.2. Let $X$ be a compact Hausdorff space. If the condition (a) of Theorem 1.1 holds, then $X$ is almost locally connected.

Proof. Assume that (a) holds and suppose that $X$ is not almost locally connected. By definition, $X$ contains mutually disjoint connected closed subsets $C_{n}(n \in \mathbf{N})$, which are open in $\overline{\cup_{n \in \mathbf{N}} C_{n}}$, the closure of $\cup_{n \in \mathbf{N}} C_{n}$ in $X$, with the following property: to each $n \in \mathbf{N}$ there correspond $x_{n}, y_{n} \in C_{n}$ such that $\left\{x_{n}\right\}_{n \in \mathbf{N}}$ and $\left\{y_{n}\right\}_{n \in \mathbf{N}}$ converge to distinct points, say $x_{0}$ and $y_{0}$. Put $F=\cup_{n \in \mathbf{N}} C_{n}$. Since $X$ is a compact Hausdorff space, there exist open neighborhoods $A$ and $B$ of $x_{0}$ and $y_{0}$ respectively such that $\bar{A} \cap \bar{B}=\emptyset$. Let $f$ be a continuous mapping of $X$ into the interval $[-1,1]$ such that $f(x)=1$ on $\bar{A}$ and $f(x)=-1$ on $\bar{B}$. We consider the following mapping $h$ of $\bar{F}$ into $\mathbf{C}$ :

$$
h(x)= \begin{cases}f(x)+\frac{i}{n}\left(1-f^{2}(x)\right) & x \in C_{n} ; n \text { is even } \\ f(x)-\frac{i}{n}\left(1-f^{2}(x)\right) & x \in C_{n} ; n \text { is odd } \\ f(x) & x \in \bar{F} \backslash F .\end{cases}
$$

We see that $h \in C(\bar{F})$. Let $\tilde{h} \in C(X)$ be a mapping with $\left.\tilde{h}\right|_{\bar{F}}=h$. Since the condition (a) of Theorem 1.1 is assumed to hold, there exist a continuous mapping $\tilde{g} \in C(X)$ and $p, q \in \mathbf{N}$ with $q / p \notin \mathbf{N}$ such that $\tilde{h}^{q}=\tilde{g}^{p}$ on $X$. Put $q=s p+r$, where $s$ and $r$ are integers with $1 \leq r \leq p-1$ (note $q / p \notin \mathbf{N}$ ). Now we define the mapping $g$ of $\bar{F}$ into $\mathbf{C}$ as follows:

$$
g(x)= \begin{cases}\frac{\tilde{g}(x)}{h^{s}(x)} & x \in \bar{F}, h(x) \neq 0 \\ 0 & x \in \bar{F}, h(x)=0 .\end{cases}
$$

Recall that $\left.\tilde{h}\right|_{\bar{F}}=h$. Since $\tilde{h}^{q}=\tilde{g}^{p}$ on $X$, for each $x \in \bar{F}$ with $h(x) \neq 0$ we obtain

$$
g^{p}(x)=\left(\frac{\tilde{g}(x)}{h^{s}(x)}\right)^{p}=\frac{\tilde{h}^{q}(x)}{h^{s p}(x)}=h^{q-s p}(x)=h^{r}(x),
$$

and so $h^{r}(x)=g^{p}(x)$ whenever $x \in \bar{F}, h(x) \neq 0$. It follows that $g \in C(\bar{F})$ such that $h^{r}=g^{p}$ on $\bar{F}$.

Pick an $n \in \mathbf{N}$ arbitrarily. By the definition of $h$, there is a continuous mapping $\theta_{n}$ of $C_{n}$ such that $h(x)=|h(x)| \exp \left(i \theta_{n}(x)\right)$ for every $x \in C_{n}$ and that $\theta_{n}\left(C_{n}\right) \subset[0, \pi]$ if $n$ is even and $\theta_{n}\left(C_{n}\right) \subset[-\pi, 0]$ if $n$ is odd. Since $h^{r}=g^{p}$ on $\bar{F}$, for each $x \in C_{n}$

$$
g^{p}(x)=|h(x)|^{r} \exp \left(\operatorname{ir} \theta_{n}(x)\right)
$$


and so there is a $p$-th root $\omega_{n}(x)$ of 1 such that

$$
g(x)=\omega_{n}(x)|h(x)|^{r / p} \exp \left(\frac{\operatorname{ir} \theta_{n}(x)}{p}\right) .
$$

Since $h, g$ and $\theta_{n}$ are continuous, $\omega_{n}(x)$ is a continuous mapping of $C_{n}$ into the set of all $p$-th roots of 1 . Furthermore, since $C_{n}$ is connected, $\omega_{n}(x)$ must be constant, say $\omega_{n}$. So,

$$
g(x)=\omega_{n}|h(x)|^{r / p} \exp \left(\frac{\operatorname{ir} \theta_{n}(x)}{p}\right) \quad\left(x \in C_{n}\right) .
$$

Since $\left\{x_{n}\right\}_{n \in \mathbf{N}}$ and $\left\{y_{n}\right\}_{n \in \mathbf{N}}$ converge to $x_{0} \in A$ and $y_{0} \in B$, respectively, we may assume that $\left\{x_{n}\right\}_{n \in \mathbf{N}} \subset A$ and $\left\{y_{n}\right\}_{n \in \mathbf{N}} \subset B$. Recall that $f=1$ on $\bar{A}$ and $f=-1$ on $\bar{B}$. So, we get $h\left(x_{n}\right)=1$ and $h\left(y_{n}\right)=-1$ for every $n \in \mathbf{N}$. Since $\theta_{2 n}\left(C_{2 n}\right) \subset[0, \pi]$ and $\theta_{2 n-1}\left(C_{2 n-1}\right) \subset$ $[-\pi, 0]$ for every $n \in \mathbf{N}$, it follows from the equation $h(x)=|h(x)| \exp \left(i \theta_{n}(x)\right)$ that $\theta_{n}\left(x_{n}\right)=$ $0, \theta_{2 n}\left(y_{2 n}\right)=\pi$ and $\theta_{2 n-1}\left(y_{2 n-1}\right)=-\pi$ for every $n \in \mathbf{N}$. It follows from (3) that $g\left(x_{n}\right)=\omega_{n}$ converges to $g\left(x_{0}\right)$. On the other hand, since $g\left(y_{n}\right)$ converges to $g\left(y_{0}\right)$, we see from (3) that both $g\left(y_{2 n}\right)=\omega_{2 n} \exp (\operatorname{ir} \pi / p)$ and $g\left(y_{2 n-1}\right)=\omega_{2 n-1} \exp (-i r \pi / p)$ converge to $g\left(y_{0}\right)$. That is,

$$
g\left(x_{0}\right) \exp \left(\frac{i r \pi}{p}\right)=g\left(y_{0}\right)=g\left(x_{0}\right) \exp \left(\frac{-i r \pi}{p}\right) .
$$

Since $\left|g\left(x_{0}\right)\right|=\left|h\left(x_{0}\right)\right|^{r / p}=\left|f\left(x_{0}\right)\right|^{r / p}=1$, we see that $\exp (\operatorname{ir} \pi / p)=\exp (-i r \pi / p)$. In other words, $r / p \in \mathbf{N}$, which contradicts $1 \leq r \leq p-1$. We thus conclude that $X$ is almost locally connected.

The following results, Lemma 2.3 and 2.4 are deduced from [13, Theorem 3.3]; Moreover, Lemma 2.4 is well-known (cf. [11, Chap.VIII $\$ 57$ Section III, Theorem 3, p.438]). Here we give a proof for the sake of completeness.

LEMMA 2.3. Let $X$ be a locally connected compact Hausdorff space. If $X$ is hereditarily unicoherent, then $\operatorname{dim} X \leq 1$.

Proof. Let $\mathfrak{A}=\left\{O_{k}\right\}_{k=1}^{n}$ be a finite open covering of $X$. We show that there is an open refinement $\mathfrak{B}$ for $\mathfrak{A}$ such that every $x \in X$ is in at most two elements of $\mathfrak{B}$. Since $X$ is assumed to be locally connected, it follows from [13, Lemma 3.2] that $X$ is an A-space, that is, the class of all open sets whose boundaries are finite sets forms an open base. Without loss of generality we may assume that each $O_{k}$ has at most finitely many boundary points. Put $B=\bigcup_{k=1}^{n}\left(\overline{O_{k}} \backslash O_{k}\right)$, where ${ }^{-}$denotes the closure in $X$. We define mutually disjoint open family $\left\{V_{k}\right\}_{k=1}^{n}$ as follows:

$$
V_{1}=O_{1} \backslash B \text { and } V_{k}=O_{k} \backslash\left(B \cup \bigcup_{j=1}^{k-1} \overline{O_{j}}\right) \text { for } k=2,3, \ldots, n
$$

Since $\left\{O_{k}\right\}_{k=1}^{n}$ is an open covering of $X$, we see that $\bigcup_{k=1}^{n} V_{k}=X \backslash B$. 
Since $B$ consists of at most finitely many points, to each $x \in B$ there corresponds an open neighborhood $U_{x}$ of $x$ with the following property: $U_{x} \subset O_{k}$ for some $k$ and $U_{x} \cap U_{y}=\emptyset$ whenever $x, y \in B, x \neq y$. Put $\mathfrak{B}=\left\{V_{k}\right\}_{k=1}^{n} \cup\left\{U_{x}: x \in B\right\}$. We see that $\mathfrak{B}$ is an open covering of $X$. Recall that both $\left\{V_{k}\right\}_{k=1}^{n}$ and $\left\{U_{x}: x \in B\right\}$ are mutually disjoint. This implies that if $x \in X$, then at most two elements of $\mathfrak{B}$ contain $x$. So, we get $\operatorname{dim} X \leq 1$.

LEMmA 2.4. Let $X$ be a locally connected compact Hausdorff space. If $X$ is hereditarily unicoherent, then $\breve{H}^{1}(X, \mathbf{Z})$ is trivial.

Proof. Assume that $X$ is hereditarily unicoherent. By a theorem of Arens and Royden, it is enough to show that the equality $C(X)^{-1}=\exp C(X)$ holds: Since $\exp C(X) \subset C(X)^{-1}$, it suffices to prove that $C(X)^{-1} \subset \exp C(X)$. To do this, pick $f \in C(X)^{-1}$ arbitrarily. Since $X$ is locally connected, each connected component of $X$ is open. It follows that $X$ has at most finitely many connected components. Without loss of generality, we may assume that $X$ is connected. Recall that $f \in C(X)^{-1}$, and so $f$ vanishes nowhere. Since $X$ is locally connected, for each $x$ in $X$ there exists a connected open neighborhood $V_{x}$ of $x$ and a continuous mapping $g_{x}$ of the closure $\overline{V_{x}}$ of $V_{x}$ into $\mathbf{C}$ such that $f=e^{g_{x}}$ on $\overline{V_{x}}$. Since $X$ is compact, there are finite number of points $x_{1}, x_{2}, \ldots, x_{n+1}$ such that $\cup_{k=1}^{n+1} V_{x_{k}}=X$. For simplicity, we denote $g_{k}=g_{x_{k}}$ and $V_{k}=V_{x_{k}}$ for $k=1,2, \ldots, n+1$. Note that $\left\{{\overline{V_{k}}}_{k=1}^{n+1}\right.$ is a class of non-empty connected closed sets with $\cup_{k=1}^{n+1} \overline{V_{k}}=X$. Since $X$ is connected, $\overline{V_{1}}$ intersects at least one of $\overline{V_{2}}, \overline{V_{3}}, \ldots, \overline{V_{n+1}}$; we may assume that $\overline{V_{1}}$ meets $\overline{V_{2}}$. Then $e^{g_{1}}=f=$ $e^{g_{2}}$ on $\overline{V_{1}} \cap \overline{V_{2}}$, and so we have $e^{g_{1}-g_{2}}=1$ on $\overline{V_{1}} \cap \overline{V_{2}}$. Since $X$ is hereditarily unicoherent, $\overline{V_{1}} \cap \overline{V_{2}}$ is connected. Hence by the continuity of $g_{1}-g_{2}$, the equation $e^{g_{1}-g_{2}}=1$ implies the existence of an integer $k_{1}$ such that

$$
g_{1}-g_{2}=2 k_{1} \pi i \quad \text { on } \quad \overline{V_{1}} \cap \overline{V_{2}} .
$$

We define a mapping $\widetilde{g_{1}}$ of $\overline{V_{1}} \cup \overline{V_{2}}$ into $\mathbf{C}$ as follows:

$$
\widetilde{g_{1}}(x)= \begin{cases}g_{1}(x) & x \in \overline{V_{1}} \\ g_{2}(x)+2 k_{1} \pi i & x \in \overline{V_{2}} \backslash \overline{V_{1}}\end{cases}
$$

It is easy to see that $\widetilde{g_{1}}$ is continuous on $\overline{V_{1}} \cup \overline{V_{2}}$ and

$$
f=e^{\widetilde{g_{1}}} \quad \text { on } \quad \overline{V_{1}} \cup \overline{V_{2}} .
$$

In the same way, $\overline{V_{1}} \cup \overline{V_{2}}$ intersects at least one of $\overline{V_{3}}, \overline{V_{4}}, \ldots, \overline{V_{n+1}}$. We may assume that $\overline{V_{1}} \cup \overline{V_{2}}$ meets $\overline{V_{3}}$. The equation $e^{\widetilde{g_{1}}}=f=e^{g_{3}}$ holds on $\left(\overline{V_{1}} \cup \overline{V_{2}}\right) \cap \overline{V_{3}}$, and so $e^{\widetilde{g_{1}}-g_{3}}=1$ on $\left(\overline{V_{1}} \cup \overline{V_{2}}\right) \cap \overline{V_{3}}$. Since $X$ is hereditarily unicoherent, $\left(\overline{V_{1}} \cup \overline{V_{2}}\right) \cap \overline{V_{3}}$ is connected. Hence by the continuity of $\widetilde{g}_{1}-g_{3}$, there exists an integer $k_{2}$ such that

$$
\widetilde{g_{1}}-g_{3}=2 k_{2} \pi i \quad \text { on } \quad\left(\overline{V_{1}} \cup \overline{V_{2}}\right) \cap \overline{V_{3}} .
$$


We define a mapping $\widetilde{g_{2}}$ of $\left(\overline{V_{1}} \cup \overline{V_{2}}\right) \cup \overline{V_{3}}$ into $\mathbf{C}$ as follows: If $x$ is in $\overline{V_{1}} \cup \overline{V_{2}}$, let $\widetilde{g_{2}}(x)=\widetilde{g_{1}}(x)$, and let $\widetilde{g_{2}}(x)=g_{3}(x)+2 k_{2} \pi i$ otherwise. It is easy to see that $\widetilde{g_{2}}$ is continuous on $\overline{V_{1}} \cup \overline{V_{2}} \cup \overline{V_{3}}$ and

$$
f=e^{\widetilde{g_{2}}} \quad \text { on } \quad \overline{V_{1}} \cup \overline{V_{2}} \cup \overline{V_{3}} .
$$

Continuing this process, we have a continuous mapping $\widetilde{g_{n}}$ of $\cup_{k=1}^{n+1} \overline{V_{k}}$ such that

$$
f=e^{\widetilde{g_{n}}} \quad \text { on } \quad \bigcup_{k=1}^{n+1} \overline{V_{k}}
$$

Since $\cup_{k=1}^{n+1} \overline{V_{k}}=X$, we have that $f \in \exp C(X)$. Since $f \in C(X)^{-1}$ was arbitrary, we conclude that $C(X)^{-1} \subset \exp C(X)$ and the proof is complete.

Lemma 2.5. Let $X$ be a compact Hausdorff space. If $\operatorname{dim} X \leq 1$ and $\check{H}^{1}(X ; \mathbf{Z})$ is trivial, then $\left\{g^{p}: g \in C(X)\right\}$ is uniformly dense in $C(X)$ for every $p \in \mathbf{N}$.

Proof. Pick $p \in \mathbf{N}$ and $f \in C(X)$ arbitrarily. We show that for every $\varepsilon>0$ there exists a $g \in C(X)$ such that $\left\|f-g^{p}\right\|_{\infty}<\varepsilon$. Without loss of generality we may assume that $\|f\|_{\infty} \leq 1$. Choose a $k \in \mathbf{N}$ so that $2^{p} / \varepsilon^{p}<k$. Then put

$$
E_{k}=\left\{x \in X:|f(x)| \geq \frac{1}{k}\right\} .
$$

Since $\operatorname{dim} X \leq 1$, there exists a $u \in C(X)^{-1}$ with $|u|=1$ on $X$ such that $u=f /|f|$ on $E_{k}$. Then $\tilde{u}(x)=\max \{|f(x)|, 1 / k\} u(x)$ is in $C(X)^{-1}$ with $\tilde{u} \mid=f$ on $E_{k}$. Since $\check{H}^{1}(X ; \mathbf{Z})$ is trivial, by a theorem of Arens and Royden there exists a $v \in \exp C(X)$ such that $\tilde{u}=v^{p}$. We define mappings $g$ and $h$ as follows:

$$
\begin{aligned}
& g(x)=\frac{\sqrt[p]{|f(x)| v(x)}}{|v(x)|} \quad(x \in X), \\
& h(x)= \begin{cases}0 & f(x)=0 \\
\frac{f(x)}{g(x)^{p-1}} & f(x) \neq 0 .\end{cases}
\end{aligned}
$$

Then we see that $g, h \in C(X),\|g\|_{\infty} \leq 1$ and $f=g^{p-1} h$. Since $f(=\tilde{u})=v^{p}$ on $E_{k}$, we see that $g=v=h$ on $E_{k}$. Therefore

$$
\begin{aligned}
\|g-h\|_{\infty} & =\sup \left\{|g(x)-h(x)|: x \in X \backslash E_{k}\right\} \\
& \leq 2 \sup \left\{\sqrt[p]{|f(x)|}: x \in X \backslash E_{k}\right\} \leq 2\left(\frac{1}{k}\right)^{1 / p}<\varepsilon .
\end{aligned}
$$

Since $f=g^{p-1} h$ and $\|g\|_{\infty} \leq 1$, it follows that

$$
\left\|f-g^{p}\right\|_{\infty}=\left\|g^{p-1} h-g^{p}\right\|_{\infty} \leq\left\|g^{p-1}\right\|_{\infty}\|h-g\|_{\infty}<\varepsilon .
$$


This completes the proof.

The case where $p=2$ in Lemma 2.6 was essentially proved in [1, Corollary 5.9]. Here, we generalize the result to the case where $p \geq 2$.

LEMmA 2.6. Let $X$ be a locally connected compact Hausdorff space and $p \in \mathbf{N}$ with $p \geq 2$. If $\left\{f_{n}{ }^{p}\right\}_{n \in \mathbf{N}} \subset C(X)$ converges uniformly to $f \in C(X)$, then there is a Cauchy subsequence of $\left\{f_{n}\right\}_{n \in \mathbf{N}}$.

Proof. For each $k \in \mathbf{N}$, set

$$
E(k)=\left\{x \in X:|f(x)|>\frac{1}{k}\right\} .
$$

Note that the closure $\overline{E(k)}$ of $E(k)$ in $X$ is a compact subset of $E(2 k)$. Since $X$ is locally connected, each connected component of $E(2 k)$ is open. So, there are finitely many connected components $C(k, 1), C(k, 2), \ldots, C\left(k, N_{k}\right)$ such that $C(k, j) \cap E(k) \neq \emptyset$ for each $j, 1 \leq$ $j \leq N_{k}$ and that

$$
E(k) \subset \bigcup_{j=1}^{N_{k}} C(k, j) \subset E(2 k) .
$$

Pick $x_{k, j} \in C(k, j) \cap E(k)$ for each $k \in \mathbf{N}$ and $j, 1 \leq j \leq N_{k}$. By a diagonal argument, we obtain a subsequence of $\left\{f_{n}\right\}_{n \in \mathbf{N}}$ converging at each point $x_{k, j}$, which we denote by the same letter $\left\{f_{n}\right\}_{n \in \mathbf{N}}$. We show that $\left\{f_{n}\right\}_{n \in \mathbf{N}}$ is a Cauchy sequence in $C(X)$. Put $\omega_{l}=\exp (2 l \pi i / p)$ for $l=0,1,2, \ldots, p-1$. Fix $k \in \mathbf{N}$ arbitrarily. We define $\varepsilon(k)$ as follows:

$$
\varepsilon(k)=\min \left\{\frac{1}{2 k}-\left(\frac{1}{2 k}\right)^{p},\left(\frac{1}{4 k}\left|\omega_{1}-1\right|\right)^{p}\right\} .
$$

Since $\lim _{n \rightarrow \infty}\left\|f_{n}{ }^{p}-f\right\|_{\infty}=0$ and since $\left\{f_{n}\right\}$ converges at each point $x_{k, j}$, we have, for a sufficiently large $n(k) \in \mathbf{N}$,

$$
\begin{aligned}
& \left\|f_{n}^{p}-f_{m}^{p}\right\|_{\infty}<\varepsilon(k) \\
& \left\|f_{n}^{p}-f\right\|_{\infty}<\varepsilon(k) \\
& \left|f_{n}\left(x_{k, j}\right)-f_{m}\left(x_{k, j}\right)\right|<\varepsilon(k)^{1 / p}
\end{aligned}
$$

for $n, m \geq n(k)$ and $j=1,2, \ldots, N_{k}$. Fix $n, m \geq n(k)$ and $x \in E(2 k)$ arbitrarily. Since

$$
f_{n}^{p}(x)-f_{m}^{p}(x)=\prod_{l=0}^{p-1}\left(f_{n}(x)-\omega_{l} f_{m}(x)\right),
$$

it follows from (6) that there exists an $l$ with $0 \leq l \leq p-1$ such that the inequality

$$
\left|f_{n}(x)-\omega_{l} f_{m}(x)\right|<\varepsilon(k)^{1 / p}
$$


holds. To prove the uniqueness of such $l$, suppose that there exists another $l^{\prime}, l \neq l^{\prime}$ such that the equation (9) is valid for $l^{\prime}$ in place of $l$. We get

$$
\begin{aligned}
\left|\omega_{l}-\omega_{l^{\prime}}\right|\left|f_{m}(x)\right| & \leq\left|\omega_{l} f_{m}(x)-f_{n}(x)\right|+\left|f_{n}(x)-\omega_{l^{\prime}} f_{m}(x)\right| \\
& <2 \varepsilon(k)^{1 / p} \leq \frac{1}{2 k}\left|\omega_{1}-1\right|,
\end{aligned}
$$

and so

$$
\left|\omega_{l}-\omega_{l^{\prime}}\right|\left|f_{m}(x)\right|<\frac{1}{2 k}\left|\omega_{1}-1\right| .
$$

On the other hand, since $x \in E(2 k)$, the inequality (7) implies that

$$
\left|f_{m}(x)\right|^{p} \geq|f(x)|-\left|f(x)-f_{m}^{p}(x)\right|>\frac{1}{2 k}-\varepsilon(k) \geq\left(\frac{1}{2 k}\right)^{p} .
$$

It follows that

$$
\left|\omega_{l}-\omega_{l^{\prime}}\right|\left|f_{m}(x)\right| \geq\left|\omega_{1}-1\right|\left|f_{m}(x)\right| \geq \frac{1}{2 k}\left|\omega_{1}-1\right|,
$$

which contradicts (10). Hence the uniqueness is proved.

Since $x \in E(2 k)$ was arbitrary, we have proved that to each $x \in E(2 k)$ there corresponds a unique $l$ such that (9) holds. This implies that if we define

$$
G_{l}(k)=\left\{x \in E(2 k):\left|f_{n}(x)-\omega_{l} f_{m}(x)\right|<\varepsilon(k)^{1 / p}\right\}
$$

for $l=0,1, \ldots, p-1$, then $\left\{G_{l}(k)\right\}_{l=0}^{p-1}$ is a mutually disjoint family with $E(2 k)=$ $\cup_{l=0}^{p-1} G_{l}(k)$. Since $G_{l}(k)$ is open for $l=0,1,2, \ldots, p-1$, each connected component of $E(2 k)$ is contained in a unique $G_{l}(k)$. By the inequality (8), we get $x_{k, j} \in G_{0}(k)$ for $j=1,2, \ldots, N_{k}$. Hence $C(k, j) \subset G_{0}(k)$ for $j=1,2, \ldots, N_{k}$. By the definition of $G_{l}(k)$, it follows from (4) that

$$
\left|f_{n}(x)-f_{m}(x)\right|<\varepsilon(k)^{1 / p}
$$

for every $x \in E(k)$. If $x \in X \backslash E(k)$, then we see from (7) that

$$
\left|f_{n}(x)\right|^{p} \leq|f(x)|+\varepsilon(k)<\frac{1}{k}+\frac{1}{2 k}<\frac{2}{k} .
$$

Thus, we have that

$$
\left|f_{n}(x)-f_{m}(x)\right| \leq\left|f_{n}(x)\right|+\left|f_{m}(x)\right|<2\left(\frac{2}{k}\right)^{1 / p}
$$

for every $x \in X \backslash E(k)$. It follows from (5), (11) and (12) that

$$
\left\|f_{n}-f_{m}\right\|_{\infty} \leq 2\left(\frac{2}{k}\right)^{1 / p}
$$

Since $k \in \mathbf{N}$ and $n, m>n(k)$ are arbitrary, $\left\{f_{n}\right\}_{n \in \mathbf{N}}$ is a Cauchy sequence in $C(X)$. 
Although Lemmas 2.7 and 2.8 are well-known (cf. [11, Chap.VIII §57 Section I, Theorem 8, p.435] and [11, Chap.VIII $§ 46$ Section XI, Theorem 2, p.165], respectively), for the sake of completeness we give a proof.

LEMMA 2.7. Let X be a compact Hausdorff space. Then the following conditions are equivalent.

(a) $\check{H}^{1}(X ; \mathbf{Z})$ is trivial.

(b) For each connected component $X_{\lambda}$ of $X, \check{H}^{1}\left(X_{\lambda} ; \mathbf{Z}\right)$ is trivial.

For a compact Hausdorff space $X$, it is well-known that

$(\sharp)$ Every connected component $X_{\lambda}$ of $X$ is the intersection of all clopen sets $G_{\mu}$ of $X$ such that $X_{\lambda} \subset G_{\mu}$.

We can prove the following as an application of $(\sharp)$.

(घ) If $O$ is open with $X_{\lambda} \subset O$ for some connected component $X_{\lambda}$ of $X$, then there is clopen $G$ such that $X_{\lambda} \subset G \subset O$.

In fact, if $G_{\mu} \supset X_{\lambda}$ is clopen with $\bigcap_{\mu \in I} G_{\mu}=X_{\lambda}$, then $\left\{X \backslash G_{\mu}\right\}_{\mu \in I}$ becomes an open covering of the closed subset $X \backslash O$, and so $X \backslash O \subset \bigcup_{i=1}^{n}\left(X \backslash G_{\mu_{i}}\right)$ for some $\mu_{1}, \mu_{2}, \ldots, \mu_{n} \in$ $I$. Then the clopen $\bigcap_{i=1}^{n} G_{\mu_{i}}$ satisfies $X_{\lambda} \subset \bigcap_{i=1}^{n} G_{\mu_{i}} \subset O$.

Proof of Lemma 2.7. First we show that (a) implies (b). Suppose that (a) is true. Let $X_{\lambda}$ be an arbitrary connected component of $X$. It is enough to show that $C\left(X_{\lambda}\right)^{-1}=$ $\exp C\left(X_{\lambda}\right)$ by a theorem of Arens-Royden. Since $\exp C\left(X_{\lambda}\right) \subset C\left(X_{\lambda}\right)^{-1}$, we show that $C\left(X_{\lambda}\right)^{-1} \subset \exp C\left(X_{\lambda}\right)$. Pick an $f \in C\left(X_{\lambda}\right)^{-1}$ arbitrary. By the Tietze extension theorem, there exists a continuous extension $\tilde{f}$ of $f$ to all of $X$. Continuity of $\tilde{f}$ implies that $\tilde{f}$ does not vanish on a certain open set $O$ that contains $X_{\lambda}$. Therefore, combining with the condition (घ), we obtain a clopen set $G$ which satisfies that $X_{\lambda} \subset G \subset O$. Now we define a mapping $\mathfrak{F}$ of $X$ into $\mathbf{C}$ as follows: Let $\mathfrak{F}(x)=\tilde{f}(x)$ if $x \in G$, and $\mathfrak{F}(x)=1$ otherwise. Then we see that $\mathfrak{F} \in C(X)^{-1}$ with $\mathfrak{F}=f$ on $X_{\lambda}$. Because $\check{H}^{1}(X ; \mathbf{Z})$ is assumed to be trivial, there exists a $g \in C(X)$ such that $\mathfrak{F}=\exp g$. It follows that $f=\exp \left(\left.g\right|_{X_{\lambda}}\right)$. Thus we see that $f \in C\left(X_{\lambda}\right)^{-1}$. Since $f$ was arbitrary, we conclude that $C\left(X_{\lambda}\right)^{-1} \subset \exp C\left(X_{\lambda}\right)$.

Next we show that (b) implies (a). Suppose that (b) is true. It is enough to show that $C(X)^{-1} \subset \exp C(X)$. Pick an $\tilde{f} \in C(X)^{-1}$ arbitrarily. Since (b) is true, to every connected component $X_{\lambda}$ of $X$, the equation $C\left(X_{\lambda}\right)^{-1}=\exp C\left(X_{\lambda}\right)$ holds. Thus to each $\lambda$, there corresponds a $g_{\lambda} \in C\left(X_{\lambda}\right)$ such that $\left.\tilde{f}\right|_{X_{\lambda}}=\exp g_{\lambda}$ holds. Let $\tilde{g}_{\lambda}$ be a continuous extension of $g_{\lambda}$ to the whole space $X$. If we put $\tilde{h}_{\lambda}=\tilde{f} / \exp \tilde{g}_{\lambda}$ on $X$, then $\tilde{h}_{\lambda}=1$ on $X_{\lambda}$. Continuity of $\tilde{h}_{\lambda}$ implies that there exists an open neighborhood $O_{\lambda} \supset X_{\lambda}$ such that $\tilde{h}_{\lambda}\left(O_{\lambda}\right) \subset\{z \in \mathbf{C}$ : $|z-1|<1 / 2\}$. Therefore, combining with $(\llbracket)$, we obtain a clopen set $G_{\lambda}$ which satisfies $X_{\lambda} \subset G_{\lambda} \subset O_{\lambda}$. Since $\tilde{h}_{\lambda}\left(G_{\lambda}\right) \subset\{z \in \mathbf{C}:|z-1|<1 / 2\}$, a continuous logarithm log of $\{z \in \mathbf{C}:|z-1|<1 / 2\}$ into $\mathbf{C}$ is well-defined. So, we get

$$
\tilde{f}=\tilde{h}_{\lambda} \exp \tilde{g}_{\lambda}=\exp \left(\tilde{g}_{\lambda}+\log \tilde{h}_{\lambda}\right) \quad \text { on } \quad G_{\lambda} .
$$


Since $\left\{G_{\lambda}\right\}_{\lambda}$ is an open covering of the compact space $X$, this covering has a finite open subcovering $\left\{G_{\lambda_{k}}\right\}_{k=1}^{n}$. The corresponding mappings to $G_{k}$ are denoted by $\tilde{g}_{k}, \tilde{h}_{k}(k=1, \ldots, n)$. Since every member of this covering is clopen, without loss of generality, we may assume that $G_{\lambda_{k_{1}}} \cap G_{\lambda_{k_{2}}}=\emptyset\left(k_{1} \neq k_{2}\right)$. Now we define a mapping $\tilde{g}$ of $X$ into $\mathbf{C}$ as follows. If $x \in X$, then there exists a unique $k$ such that $x \in G_{k}$; Let $\tilde{g}(x)=\tilde{g}_{k}(x)+\log \tilde{h}_{k}(x)$. Then we see that $\tilde{g} \in C(X)$ and $\tilde{f}=\exp \tilde{g}$. Thus we conclude that $C(X)^{-1} \subset \exp C(X)$ and this completes the proof.

LEMMA 2.8. Let X be a compact Hausdorff space. Then the following conditions are equivalent.

(a) $\operatorname{dim} X \leq 1$.

(b) For each connected component $X_{\lambda}$ of $X, \operatorname{dim} X_{\lambda} \leq 1$.

PROOF. A proof of (a) $\Rightarrow$ (b) is elementary and omitted (cf. [14]).

Conversely, suppose that (b) is true. Let $F$ be a closed subset of $X$ and $f$ an $S^{1}$-valued continuous mapping of $F$. We show that there exists an $S^{1}$-valued continuous mapping $\tilde{f}$ on $X$ such that $\left.\tilde{f}\right|_{F}=f$. Let $X_{\lambda}$ be a connected component of $X$. Since $\operatorname{dim} X_{\lambda} \leq 1$, there exists an $S^{1}$-valued continuous extension $g_{\lambda}$ of $\left.f\right|_{F \cap X_{\lambda}}$ to $X_{\lambda}$. We define a mapping $h_{\lambda}$ of $F \cup X_{\lambda}$ into $\mathbf{C}$ as follows: Let $h_{\lambda}(x)=g_{\lambda}(x)$ if $x \in X_{\lambda}$, and $h_{\lambda}(x)=f(x)$ if $x \in F \backslash X_{\lambda}$. Then we see that $h_{\lambda}$ is an $S^{1}$-valued continuous mapping on $F \cup X_{\lambda}$ satisfying $h_{\lambda}=f$ on $F$. Let $\tilde{h}_{\lambda}$ be a continuous extension of $h_{\lambda}$ to all of $X$. By definition, $\left|\tilde{h}_{\lambda}\right|=\left|h_{\lambda}\right|=1$ on $F$. Continuity of $\tilde{h}_{\lambda}$ implies that there exists an open neighborhood $O_{\lambda}$ of $X_{\lambda}$ such that $\tilde{h}_{\lambda}$ never vanishes on $O_{\lambda}$. Therefore, combined with (দ), there exists a clopen set $G_{\lambda}$ such that $X_{\lambda} \subset G_{\lambda} \subset O_{\lambda}$. Thus $\tilde{h}_{\lambda}$ never vanishes on $G_{\lambda}$. Since $\left\{G_{\lambda}\right\}_{\lambda}$ is an open covering of the compact space $X,\left\{G_{\lambda}\right\}_{\lambda}$ has a finite subcovering $\left\{G_{\lambda_{k}}\right\}_{k=1}^{n}$ for $X$. Since every $G_{\lambda_{k}}$ is clopen, without loss of generality, we may assume that $G_{\lambda_{k_{1}}} \cap G_{\lambda_{k_{2}}}=\emptyset\left(k_{1} \neq k_{2}\right)$. Now we define a mapping $\tilde{f}$ on $X$ as follows. If $x \in X$, then there exists a unique $k$ such that $x \in G_{\lambda_{k}}$ : We put $\tilde{f}(x)=\tilde{h}_{\lambda_{k}}(x) /\left|\tilde{h}_{\lambda_{k}}(x)\right|$. Since $h_{\lambda_{k}}=f$ on $F$ for every $k$, we see that $\tilde{f}$ is an $S^{1}$-valued continuous mapping of $X$ such that $\left.\tilde{f}\right|_{F}=f$ and this completes the proof.

\section{Proof of results}

Proof of Theorem 1.1. (a) $\Rightarrow$ (b) By Lemma 2.1. (b) $\Rightarrow$ (c) By Lemma 2.3 and 2.4. (c) $\Rightarrow$ (d) By Lemma 2.5. (e) $\Rightarrow$ (a) By definition.

(d) $\Rightarrow$ (e) Suppose that $\left\{g^{p}: g \in C(X)\right\}$ is uniformly dense in $C(X)$ for every $p \in \mathbf{N}$. Pick $f \in C(X)$ and $p \in \mathbf{N}$ arbitrarily. By hypothesis, there exists a sequence $\left\{g_{n}{ }^{p}\right\}_{n \in \mathbf{N}}$ such that $g_{n}{ }^{p}$ converges to $f$ as $n \rightarrow \infty$. By Lemma 2.6, there is a Cauchy subsequence $\left\{g_{n_{j}}\right\}_{j \in \mathbf{N}}$ of $\left\{g_{n}\right\}_{n \in \mathbf{N}}$. Since $C(X)$ is complete, there exists a $g \in C(X)$ such that $g_{n_{j}}$ converges to $g$ as $j \rightarrow \infty$. It follows that $f=g^{p}$ and the proof is complete. 
REMARK. Let us consider the following two conditions.

$\left(\mathrm{d}^{\prime}\right) \quad\left\{g^{p}: g \in C(X)\right\}$ is uniformly dense in $\mathrm{C}(\mathrm{X})$ for some $p \in \mathbf{N}$ with $p \geq 2$.

(e') There exists a $p \in \mathbf{N}, p \geq 2$ with the following property: For each $f \in C(X)$ there is a $g \in C(X)$ such that $f=g^{p}$.

Then the implications (e) of Theorem $1.1 \Rightarrow\left(\mathrm{e}^{\prime}\right) \Rightarrow\left(\mathrm{d}^{\prime}\right)$ are obviously true. If, in addition, $X$ is locally connected, then $\left(\mathrm{d}^{\prime}\right)$ with Lemma 2.5 implies that every $f \in C(X)$ is the $p$-th power of a $g \in C(X)$. So, we get $\left(\mathrm{d}^{\prime}\right) \Rightarrow\left(\mathrm{e}^{\prime}\right)$. Consequently, both $\left(\mathrm{d}^{\prime}\right)$ and $\left(\mathrm{e}^{\prime}\right)$ are also equivalent to all of the conditions from (a) to (e) of Theorem 1.1 whenever $X$ is locally connected. Note that Kawamura and Miura [10, Theorem 1.3] proved that if $X$ is a compact Hausdorff space with $\operatorname{dim} X \leq 1$, then the condition $\left(\mathrm{d}^{\prime}\right)$ above is equivalent to that $\check{H}^{1}(X ; \mathbf{Z})$ is $p$-divisible.

It is well-known [13, Theorem 3.3] that if $X$ is locally connected, then $C(X)$ is algebraically closed if and only if $C(X)$ is square root closed as is stated in the following theorem.

THEOREM A ([13]). Let X be a locally connected compact Hausdorff space. Then the following conditions are equivalent.

(1) $C(X)$ is algebraically closed.

(2) $C(X)$ is square-root closed.

(3) $\operatorname{dim} X \leq 1$ and $\check{H}^{1}(X ; \mathbf{Z})$ is trivial.

(4) $X$ is hereditarily unicoherent.

ProOf OF COROLlary 1.2. This is just an application of Theorem 1.1 and Theorem A.

If $X$ is first-countable, then we see that the condition (a) of Theorem 1.1 holds if and only if $C(X)$ is algebraically closed. To prove this, we need the following result, which was essentially proved by Countryman, Jr. [3] (see also [13]).

THEOREM B $([3,13])$. Let $X$ be a first-countable compact Hausdorff space. Then the following conditions are equivalent.

(1) $C(X)$ is algebraically closed.

(2) $C(X)$ is square-root closed.

(3) $X$ is almost locally connected and hereditarily unicoherent.

(4) $X$ is almost locally connected and for every connected component $X_{\lambda}$ of $X, X_{\lambda}$ is locally connected, $\operatorname{dim} X_{\lambda} \leq 1$ and $\check{H}^{1}\left(X_{\lambda} ; \mathbf{Z}\right)$ is trivial.

Proof OF Corollary 1.3. (b) $\Leftrightarrow$ (d) $\Leftrightarrow$ (e): By Theorem B, each of the conditions (b), (d) and (e) implies the other.

(a) $\Rightarrow$ (b): It follows from Lemmas 2.1 and 2.2 that (a) implies (b).

(e) $\Rightarrow$ (a): It is obvious that (e) implies (a).

Finally, we show that (c) is equivalent to the condition (4) of Theorem B. It follows from Lemmas 2.7 and 2.8 that (4) of Theorem B implies (c). Conversely, we prove that (c) implies (4) of Theorem B. By [3, Proof of Lemma 2.5], we see that each connected component $X_{\lambda}$ of 
$X$ is locally connected. It follows from Lemmas 2.7 and 2.8 that (c) implies (4) of Theorem $\mathrm{B}$, and the proof is complete.

ACKNOWLEDGEMENT. The authors would like to thank the referee for the careful reading and useful suggestions which improve the paper.

\section{References}

[ 1 ] R. B. BurCKel, Characterizations of C (X) among its subalgebras, Marcel Decker, N. Y., 1972.

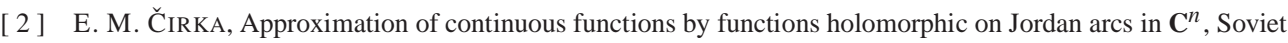
Math. 7 (1966), 336-338.

[ 3 ] R. S. Countryman JR., On the characterization of compact Hausdorff $X$ for which $C(X)$ is algebraically closed, Pacific J. Math. 20 (1967), 433-448.

[ 4 ] D. DECKARD and C. PEARCY, On matrices over the ring of continuous complex valued functions on a Stonian space, Proc. Amer. Math. Soc. 14 (1963), 322-328.

[ 5 ] D. DeckARD and C. PeArCy, On algebraic closure in function algebras, Proc. Amer. Math. Soc. 15 (1964), 259-263.

[6] T. W. Gamelin, Uniform algebras, Prentice-Hall, N. J., 1969.

[7] E. A. Gorin and M. I. KARAHANJAN, Some certain characteristic properties of the algebra of all continuous functions on a locally connected compactum, Izv. Akad. Nauk Armjan. SSR Ser. Mat. 11 (1976), 237-255.

[ 8 ] O. HATORI and T. MiURA, On a characterization of the maximal ideal spaces of commutative $C^{*}$-algebras in which every element is the square of another, Proc. Amer. Math. Soc. 128 (2000), 1185-1189.

[9] M. I. KARAHANJAN, On some algebraic characteristics of the algebra of all continuous functions on a locally connected compactum, Math. USSR-Sb. 35 (1979), 681-696.

[10] K. KAWAMURA and T. MiURA, On the existence of continuous (approximate) roots of algebraic equations, Topology Appl. 154 (2007), no. 2, 434-442.

[11] K. KURATOWs KI, Topology vol.II, New edition, Academic Press, 1968.

[12] T. MiURA, On commutative $C^{*}$-algebras in which every element is almost the square of another, Contemp. Math., 232 (1999), 239-242.

[13] T. MiURA and K. NiIJIMA, On a characterization of the maximal ideal spaces of algebraically closed commutative $C^{*}$-algebras., Proc. Amer. Math. Soc. 131 (2003), 2869-2876.

[14] K. MoritA, Dimension of general topological spaces, Surveys in general topology (G. M. REED ed.), Academic Press N.Y., 1980.

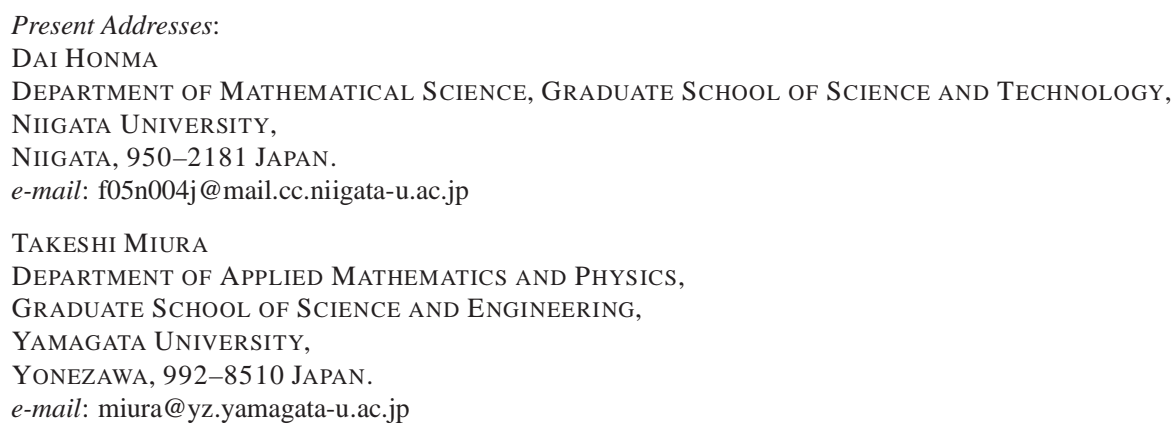

